PROCEEDINGS OF THE

AMERICAN MATHEMATICAL SOCIETY

Volume 126, Number 1, January 1998, Pages 181-187

S 0002-9939(98)03993-8

\title{
DERIVATIONS MAPPING INTO THE SOCLE, II
}

\author{
MATEJ BREŠAR
}

(Communicated by Palle E. T. Jorgensen)

\begin{abstract}
Let $\mathcal{A}$ be a unital Banach algebra. Several characterizations of continuous derivations of $\mathcal{A}$ having only elements with finite spectrum in their ranges are obtained. In particular, if $\mathcal{A}$ is semisimple, then such derivations map $\mathcal{A}$ into its socle.
\end{abstract}

\section{INTRODUCTION}

In a recent paper [4] several characterizations of inner derivations of Banach algebras having only elements with finite spectrum in their ranges were obtained. The goal of this paper is to extend these to general (continuous) derivations. In particular, we will show that the following three conditions are equivalent for a continuous derivation $d$ on a unital semisimple Banach algebra $\mathcal{A}$ :

(a) $d(x)$ has a finite spectrum for every $x \in \mathcal{A}$;

(b) $d(x)$ is algebraic for every $x \in \mathcal{A}$;

(c) $d(x)$ lies in the socle of $\mathcal{A}$ for every $x \in \mathcal{A}$.

Our main result, Theorem 3.2, is somewhat more general. We remark that implications $(\mathrm{c}) \Rightarrow(\mathrm{b}) \Rightarrow(\mathrm{a})$ are trivial since every element in the socle is algebraic, and every algebraic element has a finite spectrum. The bulk of the paper will be devoted to the proof of $(\mathrm{a}) \Rightarrow(\mathrm{c})$.

We refer to [4] for an explanation of the motivation for studying conditions (a), (b), and (c).

When considering continuous derivations of a Banach algebra $\mathcal{A}$ one often applies the well-known fact that these maps leave primitive ideals of $\mathcal{A}$ invariant, and by considering irreducible representations one thereby arrives at derivations of algebras acting densely on Banach spaces. It seems quite obvious that it is much easier to use density when dealing with inner derivations rather than with general ones. We believe that this is the reason why the proofs in the present paper are quite more involved than the proofs in [4], where only inner derivations are treated.

The main result is proved in Section 3. In Section 2 we obtain some preliminary results which (especially Theorem 2.3) might be of independent interest. This section is almost entirely algebraic - except for the last few lines we consider only outer derivations of algebras acting densely on a vector space over an arbitrary field. We should mention that in this paper we use an approach similar to one used

Received by the editors March 20, 1996 and, in revised form, July 8, 1996.

1991 Mathematics Subject Classification. Primary 46H05.

The author was supported in part by a grant from the Ministry of Science of Slovenia.

(C)1998 American Mathematical Society 
in recent papers $[2,3]$, in which in some places outer derivations are also treated separately.

\section{OUter DERIVATIONS OF DENSE ALGEBRAS}

Throughout this section, $X$ will be a vector space over a field $F, \mathcal{A}$ will be an algebra of linear operators on $X$ acting densely on $X$, and $d$ will be a derivation of $\mathcal{A}$ (i.e., a linear map of $\mathcal{A}$ into itself satisfying $d(x y)=d(x) y+x d(y)$ for all $x, y \in \mathcal{A})$. We shall say that $d$ is inner if there is a linear operator $a$ on $X$ such that $d(x)=[x, a]$ for every $x \in \mathcal{A}$ (we do not require that $a$ lies in $\mathcal{A}$ ). If $d$ is not inner, it will be called outer.

Our approach is based on the following two results:

(I) If there exist linearly independent vectors $\xi_{1}, \xi_{2} \in X$ such that $x \xi_{1}=d(x) \xi_{1}=$ 0 with $x \in \mathcal{A}$ implies $x \xi_{2}=0$, then $d$ is inner;

(II) if there exists a nonzero $\xi \in X$ such that $x \xi=0$ with $x \in \mathcal{A}$ implies $d(x) \xi=0$, then $d$ is inner.

The first result is due to Sinclair and it even characterizes inner derivations [7, Theorem 3.3]. The second result is easier to prove (see, e.g., the proofs of [2, Step $5]$ or [3, Lemma 2.3]).

We first slightly sharpen (I) and (II).

Lemma 2.1. Suppose that $d$ is outer. If $\xi_{1}, \xi_{2}$ are linearly independent vectors in $X$ and $\eta$ is any vector in $X$, then there is $x \in \mathcal{A}$ such that $x \xi_{1}=d(x) \xi_{1}=0$ and $x \xi_{2}=\eta$.

Proof. According to (I) there is $x_{0} \in \mathcal{A}$ such that $x_{0} \xi_{1}=d\left(x_{0}\right) \xi_{1}=0$ and $x_{0} \xi_{2} \neq 0$. By density, there is $x_{1} \in \mathcal{A}$ satisfying $x_{1} x_{0} \xi_{2}=\eta$. But then $x=x_{1} x_{0}$ has the desired properties.

Using (II) one similarly establishes the next lemma.

Lemma 2.2. Suppose that $d$ is outer. If $\xi, \eta \in X$ and $\xi \neq 0$, then there is $x \in \mathcal{A}$ such that $x \xi=0$ and $d(x) \xi=\eta$.

In the next theorem we generalize both lemmas.

Theorem 2.3. Suppose that $d$ is outer. If $\xi_{1}, \xi_{2}$ are linearly independent vectors in $X$ and $\eta_{1}, \eta_{2}, \eta_{3}, \eta_{4}$ are any vectors in $X$, then there is $x \in \mathcal{A}$ such that

$$
\begin{aligned}
& x \xi_{1}=\eta_{1}, d(x) \xi_{1}=\eta_{2}, \\
& x \xi_{2}=\eta_{3}, d(x) \xi_{2}=\eta_{4} .
\end{aligned}
$$

Proof. There is no loss of generality in assuming that $\eta_{1}=\eta_{2}=0$. Namely, if the theorem holds true whenever $\eta_{1}=\eta_{2}=0$ (or, analogously, $\eta_{3}=\eta_{4}=0$ ), then, given any $\eta_{i} \in X, i=1,2,3,4$, there are $x^{\prime}, x^{\prime \prime} \in \mathcal{A}$ such that

$$
\begin{gathered}
x^{\prime} \xi_{1}=d\left(x^{\prime}\right) \xi_{1}=0, x^{\prime} \xi_{2}=\eta_{3}, d\left(x^{\prime}\right) \xi_{3}=\eta_{4}, \\
x^{\prime \prime} \xi_{1}=\eta_{1}, d\left(x^{\prime \prime}\right) \xi_{1}=\eta_{2}, x^{\prime \prime} \xi_{2}=d\left(x^{\prime \prime}\right) \xi_{2}=0 .
\end{gathered}
$$

But then $x=x^{\prime}+x^{\prime \prime}$ satisfies the conclusion of the theorem. Thus, assume that $\eta_{1}=\eta_{2}=0$.

Similarly, without loss of generality we may assume that $\eta_{3} \neq 0$, for knowing that the theorem holds under this assumption we can find $x_{1}, x_{2} \in \mathcal{A}$ which, for 
instance, satisfy

$$
\begin{aligned}
& x_{1} \xi_{1}=0, d\left(x_{1}\right) \xi_{1}=0, x_{1} \xi_{2}=\xi_{2}, d\left(x_{1}\right) \xi_{2}=\eta_{4}, \\
& x_{2} \xi_{1}=0, d\left(x_{2}\right) \xi_{1}=0, x_{2} \xi_{2}=-\xi_{2}, d\left(x_{2}\right) \xi_{2}=0,
\end{aligned}
$$

so that $x=x_{1}+x_{2}$ satisfies $x \xi_{1}=d(x) \xi_{1}=x \xi_{2}=0, d(x) \xi_{2}=\eta_{4}$. Thus, let $\eta_{3} \neq 0$.

By Lemma 2.1 there is $x_{0} \in \mathcal{A}$ such that

$$
x_{0} \xi_{1}=0, d\left(x_{0}\right) \xi_{1}=0, x_{0} \xi_{2}=\eta_{3} .
$$

Let $\mathcal{L}$ be a left ideal generated by $x_{0}$. Clearly, every element $x$ in $\mathcal{L}$ satisfies $x \xi_{1}=d(x) \xi_{1}=0$. Therefore, it suffices to prove that there is an $x \in \mathcal{L}$ satisfying $x \xi_{2}=\eta_{3}$ and $d(x) \xi_{2}=\eta_{4}$.

Suppose first that the vectors $d\left(x_{0}\right) \xi_{2}$ and $\eta_{3}$ are linearly dependent, that is, $d\left(x_{0}\right) \xi_{2}=\alpha \eta_{3}$ for some $\alpha \in F$. Applying Lemma 2.2 we see that there is a $y_{0} \in \mathcal{A}$ satisfying

$$
y_{0} \eta_{3}=0, d\left(y_{0}\right) \eta_{3}=\eta_{4}-\alpha \eta_{3} .
$$

Hence $x=y_{0} x_{0}+x_{0} \in \mathcal{L}$ satisfies

$$
\begin{gathered}
x \xi_{2}=y_{0} \eta_{3}+\eta_{3}=\eta_{3}, \\
d(x) \xi_{2}=d\left(y_{0}\right) x_{0} \xi_{2}+y_{0} d\left(x_{0}\right) \xi_{2}+d\left(x_{0}\right) \xi_{2} \\
=d\left(y_{0}\right) \eta_{3}+y_{0}\left(\alpha \eta_{3}\right)+\alpha \eta_{3}=\eta_{4} .
\end{gathered}
$$

Thus, the theorem is proved when this situation occurs.

Assume, therefore, that $d\left(x_{0}\right) \xi_{2}$ and $\eta_{3}$ are linearly independent. Using Lemma 2.1 again we get an element $y_{1} \in \mathcal{A}$ satisfying

$$
y_{1} \eta_{3}=d\left(y_{1}\right) \eta_{3}=0, y_{1} d\left(x_{0}\right) \xi_{2}=\eta_{4}-d\left(x_{0}\right) \xi_{2} .
$$

Consequently, setting $x=y_{1} x_{0}+x_{0} \in \mathcal{L}$ we have

$$
\begin{gathered}
x \xi_{2}=y_{1} \eta_{3}+\eta_{3}=\eta_{3}, \\
d(x) \xi_{2}=d\left(y_{1}\right) x_{0} \xi_{2}+y_{1} d\left(x_{0}\right) \xi_{2}+d\left(x_{0}\right) \xi_{2} \\
=d\left(y_{1}\right) \eta_{3}+\eta_{4}-d\left(x_{0}\right) \xi_{2}+d\left(x_{0}\right) \xi_{2}=\eta_{4} .
\end{gathered}
$$

The proof is now complete.

It seems reasonable to conjecture that the theorem can be extended to the situation where we have $n>2$ linearly independent vectors $\xi_{1}, \ldots, \xi_{n}$. We leave this as an open problem.

Lemma 2.4. Suppose that $d$ is outer. Let $\xi, \xi_{1}, \ldots, \xi_{k}$ be linearly independent vectors in $X$ and $\eta \in X$. Then there is $x \in \mathcal{A}$ such that

$$
\begin{gathered}
x \xi=\eta, d(x) \xi=0, \\
x \xi_{1}=x \xi_{2}=\cdots=x \xi_{k}=0 .
\end{gathered}
$$

Proof. Pick $x_{0} \in \mathcal{A}$ such that $x_{0} \xi=\xi$ and $x_{0} \xi_{i}=0$.

Suppose first that $d\left(x_{0}\right) \xi$ and $\xi$ are linearly independent. Applying Theorem 2.3 we see that there is $y \in \mathcal{A}$ such that $y \xi=\eta, d(y) \xi=0$ and $y d\left(x_{0}\right) \xi=0$. It is immediate then that $x=y x_{0}$ has the desired properties.

Assume, therefore, that $d\left(x_{0}\right) \xi=\alpha \xi$ for some $\alpha \in F$. Using Theorem 2.3 again we can find $z \in \mathcal{A}$ such that $z \xi=\eta$ and $d(z) \xi=-\alpha \eta$. But then $x=z x_{0}$ is the right element. 
Let us point out that the next lemma holds for all, not necessarily outer, derivations. However, in the case of inner derivations we have to assume that $F=\mathbb{C}$. In the course of the proof we shall apply the following result due to Amitsur [1, Lemma 1]: if $T_{1}, \ldots, T_{n}$ are linear maps such that for every vector $\xi$, the vectors $T_{1} \xi, \ldots, T_{n} \xi$ are linearly dependent modulo some (fixed) finite dimensional subspace $U_{0}$, then some nontrivial linear combination of the $T_{i}$ 's is an operator of finite rank.

Lemma 2.5. Let $F=\mathbb{C}$. Suppose that d does not map $\mathcal{A}$ into the algebra of finite rank operators. Then for every $m \in \mathbb{N}$ there exist $x \in \mathcal{A}$ and linearly independent vectors $\xi_{1}, \ldots, \xi_{m} \in X$ such that

$$
x \xi_{j}=0, d(x) \xi_{j}=j \xi_{j}, \quad j=1, \ldots, m .
$$

Proof. We first treat the case when $d$ is outer. Proceed by induction on $m$. If $m=1$, then, in view of Lemma 2.2 , we can choose any nonzero $\xi_{1} \in X$.

Thus, let $m>1$. By the induction assumption, there exist linearly independent $\xi_{1}, \ldots, \xi_{m-1} \in X$ and $x_{0} \in \mathcal{A}$ such that

$$
x_{0} \xi_{j}=0, d\left(x_{0}\right) \xi_{j}=j \xi_{j}, \quad j=1, \ldots, m-1 .
$$

As $x_{0}=0$ certainly implies $d\left(x_{0}\right)=0$, these identities show that $x_{0}$ and $d\left(x_{0}\right)$ are linearly independent operators.

Assume first that the vectors $x_{0} \xi, d\left(x_{0}\right) \xi, \xi_{1}, \ldots, \xi_{m-1}$ are linearly dependent for every $\xi \in X$. This means that $x_{0} \xi$ and $d\left(x_{0}\right) \xi$ are linearly dependent modulo $U_{0}$, the subspace of $X$ generated by $\xi_{1}, \ldots, \xi_{m-1}$. By a result of Amitsur stated above it follows that some nontrivial linear combination of $x_{0}$ and $d\left(x_{0}\right)$ is a finite rank operator; it is certainly a nonzero one since $x_{0}$ and $d\left(x_{0}\right)$ are independent. Thus, $\mathcal{A}$ contains nonzero finite rank operators. But then every derivation of $\mathcal{A}$ is inner (see, e.g., [5, Theorem 3, p. 87] or [7, Remark 3.5]), contrary to the assumption.

Therefore, we may assume that there is a vector in $X$, which we denote by $\xi_{m}$, such that $\zeta_{1}=x_{0} \xi_{m}, \zeta_{2}=d\left(x_{0}\right) \xi_{m}, \xi_{1}, \ldots, \xi_{m-1}$ are linearly independent vectors. Then $\xi_{1}, \ldots, \xi_{m-1}, \xi_{m}$ are linearly independent too. Namely, assuming that they are dependent it follows that $\xi_{m}$ lies in the linear span of $\xi_{1}, \ldots, \xi_{m-1}$, which further yields $\zeta_{1}=x_{0} \xi_{m}=0$. By Lemma 2.4 , there is a $y \in \mathcal{A}$ such that

$$
\begin{gathered}
y \zeta_{1}=-\zeta_{1}, d(y) \zeta_{1}=0, \\
y \xi_{1}=\cdots=y \xi_{m-1}=0, y \zeta_{2}=0 .
\end{gathered}
$$

Next, choose $z \in \mathcal{A}$ such that

$$
z \zeta_{2}=m \xi_{m}, z \xi_{j}=\xi_{j}, \quad j=1, \ldots, m-1 .
$$

Set $x=z y x_{0}+z x_{0}$ and note that

$$
\begin{gathered}
x \xi_{j}=0, \quad j=1, \ldots, m-1, \\
d(x) \xi_{j}=d(z y+z) x_{0} \xi_{j}+z y d\left(x_{0}\right) \xi_{j}+z d\left(x_{0}\right) \xi_{j} \\
=z y\left(j \xi_{j}\right)+z\left(j \xi_{j}\right)=j \xi_{j}, \quad j=1, \ldots, m-1, \\
x \xi_{m}=z y x_{0} \xi_{m}+z x_{0} \xi_{m}=z\left(y \zeta_{1}+\zeta_{1}\right)=0, \\
d(x) \xi_{m}=d(z) y x_{0} \xi_{m}+z d(y) x_{0} \xi_{m}+z y d\left(x_{0}\right) \xi_{m}+d(z) x_{0} \xi_{m}+z d\left(x_{0}\right) \xi_{m} \\
=d(z) y \zeta_{1}+z d(y) \zeta_{1}+z y \zeta_{2}+d(z) \zeta_{1}+z \zeta_{2}=m \xi_{m} .
\end{gathered}
$$


This proves the lemma for the case when $d$ is outer.

Now, assume that $d$ is inner, that is, there is a linear operator $a$ on $X$ such that $d(x)=[x, a], x \in \mathcal{A}$. It is clear that $a$ cannot be written as a sum of a scalar multiple of the identity and a finite rank operator, for otherwise $d(x)$ would have finite rank for every $x \in \mathcal{A}$. But then there exist $\xi_{1}, \ldots, \xi_{m} \in X$ such that the vectors $\xi_{1}, \ldots, \xi_{m}, a \xi_{1}, \ldots, a \xi_{m}$ are linearly independent [4, Lemma 3.1]. Pick $x \in \mathcal{A}$ such that $x \xi_{j}=0, x a \xi_{j}=j \xi_{j}, j=1, \ldots, m$. But then $d(x) \xi_{j}=x a \xi_{j}-a x \xi_{j}=j \xi_{j}$. The proof of the lemma is thereby completed.

\section{Characterizing Derivations mapping into the SOCle}

We first fix the notation. In this section, $\mathcal{A}$ denotes a complex Banach algebra. By $\operatorname{Prim}(\mathcal{A})$ we denote the set of primitive ideals of $\mathcal{A}$. We write $\sigma(x)$ for the spectrum of $x \in \mathcal{A}$, and $\sharp \sigma(x)$ for its cardinality.

Suppose that $\mathcal{A}$ is semisimple. Then the sum of all minimal left ideals of $\mathcal{A}$ coincides with the sum of all minimal right ideals of $\mathcal{A}$, and is called the socle of $\mathcal{A}$. It will be denoted by $\operatorname{soc} \mathcal{A}$. If $\mathcal{A}$ does not contain minimal one-sided ideals, we define $\operatorname{soc}(\mathcal{A})=\{0\}$.

We begin with an auxiliary result.

Lemma 3.1. Let $d$ be a continuous derivation of a Banach algebra $\mathcal{A}$. Suppose that $P_{1}, \ldots, P_{m} \in \operatorname{Prim}(\mathcal{A})\left(P_{i} \neq P_{j}\right.$ whenever $\left.i \neq j\right)$ are such that $d(\mathcal{A}) \nsubseteq P_{j}$, $j=1, \ldots, m$. Let $\pi_{1}, \ldots, \pi_{m}$ be irreducible representations of $\mathcal{A}$ on Banach spaces $X_{1}, \ldots, X_{m}$, respectively, such that $\operatorname{Ker} \pi_{j}=P_{j}, j=1, \ldots, m$. Then there exist nonzero vectors $\xi_{j} \in X_{j}, j=1, \ldots, m$, and $x \in \mathcal{A}$ such that

$$
\pi_{j}(x) \xi_{j}=0, \pi_{j}(d(x)) \xi_{j}=j \xi_{j}, \quad j=1, \ldots, m .
$$

Proof. We proceed by induction on $m$. Let $m=1$. As $d\left(P_{1}\right) \subseteq P_{1}[7$, Theorem 2.2], $d$ induces a nonzero derivation $d_{1}$ on the algebra $\mathcal{A}_{1}=\pi_{1}(\mathcal{A})$ defined by $d_{1} \pi_{1}=\pi_{1} d$. Thus, we actually have to prove the following: if $d_{1}$ is a nonzero derivation on a dense algebra of linear operators on $X_{1}$, then there exist nonzero $\xi_{1} \in X_{1}$ and $x_{1} \in \mathcal{A}_{1}$ such that $x_{1} \xi_{1}=0, d_{1}\left(x_{1}\right) \xi_{1}=\xi_{1}$. If $d$ is outer, then, by Lemma 2.2 , we can choose any nonzero $\xi_{1} \in X_{1}$. Suppose, therefore, that $d_{1}(x)=[x, a], x \in \mathcal{A}_{1}$, for some linear operator $a$ on $X_{1}$. As $d_{1} \neq 0$, there exists $\xi_{1} \in X_{1}$ such that $\xi_{1}$ and $a \xi_{1}$ are linearly independent. By density, there is $x_{1} \in \mathcal{A}_{1}$ such that $x_{1} \xi_{1}=0$ and $x_{1} a \xi_{1}=\xi_{1}$. But then $d_{1}\left(x_{1}\right) \xi_{1}=\left[x_{1}, a\right] \xi_{1}=\xi_{1}$.

Now, let $m>1$. As $P_{1}, \ldots, P_{m}$ are distinct, at least one of them, say $P_{m}$, satisfies $P_{j} \nsubseteq P_{m}, j=1, \ldots, m-1$. By the induction hypothesis, there exist $x_{0} \in \mathcal{A}$ and nonzero vectors $\xi_{j} \in X_{j}, j=1, \ldots, m-1$, such that

$$
\pi_{j}\left(x_{0}\right) \xi_{j}=0, \pi_{j}\left(d\left(x_{0}\right)\right) \xi_{j}=j \xi_{j}, \quad j=1, \ldots, m-1 .
$$

As above, we define a derivation $d_{m} \neq 0$ on $\mathcal{A}_{m}=\pi_{m}(\mathcal{A})$ by $d_{m} \pi_{m}=\pi_{m} d$. Since every primitive ideal is also a prime ideal, $P_{j} \nsubseteq P_{m}, j=1, \ldots, m-1$, yields $P_{1} \cdots P_{m-1} \nsubseteq P_{m}$, which in turn implies $P_{1} \cap \cdots \cap P_{m-1} \nsubseteq P_{m}$. Whence $\mathcal{I}=\pi_{m}\left(P_{1} \cap \cdots \cap P_{m-1}\right)$ is a nonzero ideal of $\mathcal{A}_{m}$, and therefore a dense algebra of operators on $X_{m}$ [5, Theorem 4, p. 33].

We claim that the lemma will be established by showing that there are $z \in \mathcal{I}$ and a nonzero vector $\xi_{m} \in X_{m}$ satisfying

$$
z \xi_{m}=-\pi_{m}\left(x_{0}\right) \xi_{m}, d_{m}(z) \xi_{m}=m \xi_{m}-\pi_{m}\left(d\left(x_{0}\right)\right) \xi_{m} .
$$


Suppose that such $z$ and $\xi_{m}$ exist. We have $z=\pi_{m}\left(z_{0}\right)$ for some $z_{0} \in P_{1} \cap \cdots \cap P_{m-1}$. Set $x=x_{0}+z_{0}$. Then we have

$$
\begin{aligned}
\pi_{m}(x) & \xi_{m}=\pi_{m}\left(x_{0}\right) \xi_{m}+\pi_{m}\left(z_{0}\right) \xi_{m}=\pi_{m}\left(x_{0}\right) \xi_{m}+z \xi_{m}=0, \\
\pi_{m}(d(x)) \xi_{m} & =\pi_{m}\left(d\left(x_{0}\right)\right) \xi_{m}+\pi_{m}\left(d\left(z_{0}\right)\right) \xi_{m} \\
& =\pi_{m}\left(d\left(x_{0}\right)\right) \xi_{m}+d_{m}\left(\pi_{m}\left(z_{0}\right)\right) \xi_{m}=\pi_{m}\left(d\left(x_{0}\right)\right) \xi_{m}+d_{m}(z) \xi_{m} \\
& =\pi_{m}\left(d\left(x_{0}\right)\right) \xi_{m}+m \xi_{m}-\pi_{m}\left(d\left(x_{0}\right)\right) \xi_{m}=m \xi_{m} .
\end{aligned}
$$

Since $z_{0} \in P_{1} \cap \cdots \cap P_{m-1}$, using Sinclair's result [7, Theorem 2.2] again, we see that $d\left(z_{0}\right)$ lies in $P_{1} \cap \cdots \cap P_{m-1}$, too. Consequently, $\pi_{j}\left(z_{0}\right)=\pi_{j}\left(d\left(z_{0}\right)\right)=0$, and hence $\pi_{j}(x)=\pi_{j}\left(x_{0}\right), \pi_{j}(d(x))=\pi_{j}\left(d\left(x_{0}\right)\right), j=1, \ldots, m-1$. Thus, $x$ has all the desired properties and the lemma is proved.

Now let us find such $z \in \mathcal{I}$ and $\xi_{m} \in X_{m}$. Suppose first that $d_{m}$ is inner, i.e., $d_{m}(x)=[x, a], x \in \mathcal{A}_{m}$, for some linear operator $a$ on $X_{m}$. As $d_{m} \neq 0$, there exists $\xi_{m} \in X_{m}$ such that $\xi_{m}$ and $a \xi_{m}$ are linearly independent. Recall that $\mathcal{I}$ acts densely on $X_{m}$. Thus, there is $z \in \mathcal{I}$ such that $z \xi_{m}=-\pi_{m}\left(x_{0}\right) \xi_{m}$ and $z a \xi_{m}=m \xi_{m}-\pi_{m}\left(x_{0}\right) a \xi_{m}$. But then

$$
\begin{aligned}
d_{m}(z) \xi_{m} & =[z, a] \xi_{m}=z a \xi_{m}-a z \xi_{m} \\
& =m \xi_{m}-\pi_{m}\left(x_{0}\right) a \xi_{m}+a \pi_{m}\left(x_{0}\right) \xi_{m}=m \xi_{m}-\left[\pi_{m}\left(x_{0}\right), a\right] \xi_{m} \\
& =m \xi_{m}-d_{m}\left(\pi_{m}\left(x_{0}\right)\right) \xi_{m}=m \xi_{m}-\pi_{m}\left(d\left(x_{0}\right)\right) \xi_{m} .
\end{aligned}
$$

Thus, we may assume that $d_{m}$ is outer. Since $d\left(P_{1} \cap \cdots \cap P_{m-1}\right) \subseteq P_{1} \cap \cdots \cap P_{m-1}$, we have $d_{m}(\mathcal{I}) \subseteq \mathcal{I}$. Consider $d_{m} \mid \mathcal{I}$, a restriction of $d_{m}$ to $\mathcal{I}$. We claim that this is an outer derivation of $\mathcal{I}$. Indeed, assuming that it is inner, that is, $d_{m}(u)=$ $[u, a], u \in \mathcal{I}$, for some linear operator $a$ on $X_{m}$, we see that for any $u \in \mathcal{I}$ and $x \in \mathcal{A}_{m}$ we have

$$
u d_{m}(x)=d_{m}(u x)-d_{m}(u) x=[u x, a]-[u, a] x=u[x, a] .
$$

That is, $\mathcal{I}\left(d_{m}(x)-[x, a]\right)=0$, which certainly yields $d_{m}(x)=[x, a], x \in \mathcal{A}_{m}$, contrary to the assumption. Therefore, $d_{m} \mid \mathcal{I}$ is an outer derivation of a dense algebra of linear operators on $X_{m}$. As a very special case of Theorem 2.3 we see that given any nonzero $\xi_{m} \in X_{m}$, there is a $z \in \mathcal{I}$ satisfying the desired relations $z \xi_{m}=-\pi_{m}\left(x_{0}\right) \xi_{m}$ and $d_{m}(z) \xi_{m}=m \xi_{m}-\pi_{m}\left(d\left(x_{0}\right)\right) \xi_{m}$. The proof of the lemma is now complete.

We are now in a position to prove the main result which generalizes [4, Theorem 3.2] from inner derivations to continuous derivations.

Theorem 3.2. Let $d$ be a continuous derivation of a unital Banach algebra $\mathcal{A}$. The following conditions are equivalent:

(i) $\sharp \sigma(d(x))$ is finite for every $x \in \mathcal{A}$;

(ii) there is $n \in \mathbb{N}$ such that $\sharp \sigma(d(x)) \leq n$ for every $x \in \mathcal{A}$;

(iii) $d(x)+P \in \operatorname{soc}(\mathcal{A} / P)$ for every $x \in \mathcal{A}$ and every $P \in \operatorname{Prim}(\mathcal{A})$, and $d(\mathcal{A}) \subseteq P$ for all but finitely many $P \in \operatorname{Prim}(\mathcal{A})$.

Moreover, if $\mathcal{A}$ is semisimple, then the above conditions are equivalent to the following two equivalent conditions:

(iv) $d(x)$ lies in $\operatorname{soc}(\mathcal{A})$ for every $x \in \mathcal{A}$.

(v) $d(x)$ is algebraic for every $x \in \mathcal{A}$. 
Proof. The implication (i) $\Rightarrow$ (ii) is just a special case of [4, Lemma 2.1].

Assume that (ii) is true. Pick $P \in \operatorname{Prim}(\mathcal{A})$. Let $\pi_{P}$ be an irreducible representation of $\mathcal{A}$ on a Banach space $X$ such that $\operatorname{Ker} \pi_{P}=P$. As above, define a derivation $d_{P}$ on $\pi_{P}(\mathcal{A})$ by $d_{P} \pi_{P}=\pi_{P} d$. Suppose that the range of $d_{P}$ does not lie in the algebra of finite rank operators. Then, by Lemma 2.5 , there exist $x \in \mathcal{A}$ and nonzero vectors $\xi_{1}, \ldots, \xi_{n+1} \in X$ such that $d_{P}\left(\pi_{P}(x)\right) \xi_{j}=j \xi_{j}, j=1, \ldots, n+1$. This means that $\{1,2, \ldots, n+1\} \subseteq \sigma\left(d_{P}\left(\pi_{P}(x)\right)\right)=\sigma\left(\pi_{P}(d(x))\right) \subseteq \sigma(d(x))$, contrary to the assumption. Thus, $\left.d_{P}\left(\pi_{P}(x)\right)=\pi_{P}(d(x))\right)$ has a finite rank for every $x \in \mathcal{A}$. This means that $\pi_{P}(d(x)) \in \operatorname{soc}\left(\pi_{P}(\mathcal{A})\right)$, which is equivalent to $d(x)+P \in \operatorname{soc}(\mathcal{A} / P)$. Thus, we have proved the first part of (iii). In order to prove the second part, we assume that there exist $n+1$ distinct primitive ideals $P_{1}, \ldots, P_{n+1}$ such that $d(\mathcal{A}) \nsubseteq P_{j}, j=1, \ldots, n+1$. Let $\pi_{1}, \ldots, \pi_{n+1}$ be irreducible representations of $\mathcal{A}$ on Banach spaces $X_{1}, \ldots, X_{n+1}$, respectively, such that $\operatorname{Ker} \pi_{j}=P_{j}$. According to Lemma 3.1 there are nonzero vectors $\xi_{j} \in X_{j}, j=1, \ldots, n+1$, and $x \in \mathcal{A}$ such that $\pi_{j}(d(x)) \xi_{j}=j \xi_{j}, j=1, \ldots, n+1$. Thus, again we arrive at the contradiction $\{1,2, \ldots, n+1\} \subseteq \sigma(d(x))$. This means that there exist at most $n$ primitive ideals not containing the range of $d$. The proof of the implication (ii) $\Rightarrow($ iii) is thus complete.

Recall that

$$
\sigma(d(x))=\bigcup_{P \in \operatorname{Prim}(\mathcal{A})} \sigma(d(x)+P)
$$

(this can be, for instance, deduced from [6, Theorem 2.2.9]). Therefore, assuming (iii), we have

$$
\sigma(d(x)) \subset\{0\} \cup \sigma\left(d(x)+P_{1}\right) \cup \cdots \cup \sigma\left(d(x)+P_{m}\right),
$$

where the $P_{i}$ 's are the only primitive ideals not containing the range of $d$. Also, $d(x)+P_{j}$ has a finite spectrum in $\mathcal{A} / P_{j}$ for it lies in its socle. But then (i) holds true.

We have thereby proved that (i), (ii), and (iii) are equivalent. Now assume that $\mathcal{A}$ is semisimple. By [4, Proposition 2.2] we see that (iii) $\Rightarrow$ (iv). As already mentioned in the introduction, the implications (iv) $\Rightarrow(\mathrm{v})$ and $(\mathrm{v}) \Rightarrow(\mathrm{i})$ are trivial. The proof is complete.

\section{REFERENCES}

1. S. A. Amitsur, Generalized polynomial identities and pivotal monomials, Trans. Amer. Math. Soc. 114 (1965), 210-226. MR 30:3117

2. M. Brešar, Derivations of noncommutative Banach algebras II, Arch. Math. 63 (1994), 56-59. MR 95g: 46086

3. M. Brešar, M. Mathieu, Derivations mapping into the radical, III, J. Funct. Anal. 133 (1995), 21-29. MR 96i: 46054

4. M. Brešar, P. Šemrl, Derivations mapping into the socle, Math. Proc. Cambridge Phil. Soc. 120 (1996), 339-346. MR 96m:46085

5. N. Jacobson, Structure of rings, Amer. Math. Soc. Colloq. Publ., Vol. 37, Amer. Math. Soc., Providence, R.I., 1956. MR 18:373d; MR 36:5158

6. C. E. Rickart, General theory of Banach algebras, The University Series in Higher Mathematics, van Nostrand, 1960. MR 22:5903

7. A. M. Sinclair, Continuous derivations on Banach algebras, Proc. Amer. Math. Soc. 20 (1969), 166-170. MR 38:1530

Department of Mathematics, University of Maribor, PF, KorošKa 160, 62000 MariBOR, SLOVENIA

E-mail address: bresar@uni-mb.sl 